\title{
ON CARDINALITY OF SUMSETS
}

\author{
M. Z. GARAEV and KA-LAM KUEH
}

(Received 1 January 2002; revised 23 October 2003)

Communicated by W. W. L. Chen

\begin{abstract}
In this article we study some special problems of the additive number theory connected with an estimate of cardinality of a sum of two sets, which can be convex as well as non-convex sequences.

2000 Mathematics subject classification: primary $11 \mathrm{P} 99$.

Keywords and phrases: cardinality, a sum of sets, convex sequences, non-convex sequences.
\end{abstract}

\section{Introduction}

The problem of estimating cardinality of sumsets is one of the interesting and difficult topics of the additive number theory. In this note we deal with a particular case of this problem and discuss some special questions concerning cardinality of a sum of two sets.

For a given $\alpha, 0<\alpha \leq 1$, denote $A_{\alpha}=\left\{1,2, \ldots,\left[n^{\alpha}\right]\right\}$ where $n$ is a positive integer parameter, $n>3$. Let $c$ denote a positive constant. The real valued function $f(x)$ is required to be an increasing strictly convex function of a positive integer variable $x \in A_{1}$, that is, to satisfy

$$
0<f(2)-f(1)<f(3)-f(2)<\cdots<f(n)-f(n-1) .
$$

Hegyvári [6] proved that the set

$$
f\left(A_{1}\right)-f\left(A_{1}\right)=\left\{f\left(a_{1}\right)-f\left(a_{2}\right): a_{1}, a_{2} \in A_{1}\right\}
$$

has at least $c n(\log n / \log \log n)$ elements, that is,

$$
\left|f\left(A_{1}\right)-f\left(A_{1}\right)\right|>c n \frac{\log n}{\log \log n} .
$$

(C) 2005 Australian Mathematical Society $1446-7887 / 05 \$ A 2.00+0.00$ 
Using Jarnik's result [7] on integral lattice points on a strictly convex line or a method used to prove Lemma of [3] one is able to establish that for a given $s$ the number of solutions of the equation $s=f(x)+f(y)$ is at most $c n^{2 / 3}$. This remark will allow us to note that (2) can be improved to $c n^{4 / 3}$. As it was noticed by Elekes, Nathanson and Ruzsa in [2], the third author, in his unpublished work, improved (2) to $\mathrm{Cn}^{4 / 3}$ and in [2] this result was further improved to

$$
\left|f\left(A_{1}\right) \pm f\left(A_{1}\right)\right|>c n^{3 / 2},
$$

where ' \pm ' here denotes any of the signs ' $t$ ' and ' - ', that is, the corresponding estimate takes place for the sumset and for the difference set as well. Applying the methods of combinatorial geometry they obtained more general theorem from which (3) follows together with many other significant results.

In this article, using entirely elementary method, we obtain a new result which includes estimate (3) with $c=1 / 5$.

THEOREM 1.1. Assume (1). Then $n^{3 / 2} / 5<\left|f\left(A_{1 / 2}\right) \pm f\left(A_{1}\right)\right|<n^{3 / 2}$. Further if, $0<\alpha<1 / 2$ then $\left|f\left(A_{\alpha}\right) \pm f\left(A_{1}\right)\right|=\left[n^{\alpha}\right] n+O\left(n^{3 \alpha}\right)$, where the constant implicit in the $O$ symbol is absolute.

The upper bound estimate of the first statement is trivial. Further improvement of the error term $O\left(n^{3 \alpha}\right)$ in the second estimate would be of some interest, if such one exists. We also note that our elementary argument allows us to prove that for any $\epsilon>0, n>n_{1}(\epsilon)>0$ estimate (3) holds with $c=(3 / 4)-\epsilon$.

Let us now consider special non-convex sequences. Deshouillers [1] proved that for $1<\gamma<4 / 3$ any sufficiently large positive integer is representable in a form $\left[x^{\gamma}\right]+\left[y^{\gamma}\right]$ with nonnegative integers $x, y$. Gritsenko [5] extended the range of $\gamma$ possessing this property to $1<\gamma<55 / 41$. In our work [4] we proved that if $\alpha \in(1,3 / 2]$, then there are $\gg N$ numbers $n \leq N$ representable as $n=\left[x^{\gamma}\right]+\left[y^{\gamma}\right]$. Substantial improvement in this problem was made by Konyagin [8]. He established that for any $\gamma \in(1,3 / 2)$ a sufficiently large positive integer $n$ is representable in a form $n=\left[x^{\gamma}\right]+\left[y^{\gamma}\right]$.

For $a>0,1<\gamma<2, S=S(a, \gamma, n)=\left\{\left[a x^{\gamma}\right]: x=1,2, \ldots, n\right\}$.

THEOREM 1.2. For $3 / 2<\gamma<2$ we have

$$
|S+S| \gg \underset{a . \gamma}{\gg \min }\left\{n^{(2 \gamma+1) /(\gamma+1)}, \frac{n^{\gamma}}{\log n}\right\} .
$$

COROLLARY 1.3. For $3 / 2<\gamma \leq(\sqrt{5}+1) / 2$ we have

$$
\frac{n^{\gamma}}{\log n} \underset{a, \gamma}{\ll}|S+S| \underset{a, \gamma}{\ll} n^{\gamma}
$$




\section{Proof of Theorem 1.1}

We may suppose that $n>25$.

Assuming $0<\alpha \leq 1 / 2$ we denote by $B_{\alpha}=\left\{\left[n^{\alpha}\right]+1,\left[n^{\alpha}\right]+2, \ldots, n\right\}$. Let $J=J(\alpha, n)$ be the number of solutions of the equation

$$
f(x)+f(y)=f(u)+f(v), \quad x, u \in A_{\alpha}, \quad y, v \in B_{\alpha} .
$$

For any $l, 1 \leq l<\left[n^{\alpha}\right]$, denote by $J_{l}=J_{l}(\alpha, n)$ the number of solutions of the equation

$$
f(x)+f(y)=f(x+l)+f(z), \quad x, x+l \in A_{\alpha}, \quad y, z \in B_{\alpha} .
$$

Obviously

$$
J=\left[n^{\alpha}\right]\left(n-\left[n^{\alpha}\right]\right)+2 \sum_{1 \leq l<\left[n^{\alpha}\right]} J_{l}
$$

Let us estimate $J_{l}$. From (1) and (4) it follows that $x<x+l<z<y$ and that for any $d \geq l$ we have $f(y)-f(y-d)>f(x+l)-f(x)$. Furthermore, for fixed $x \in A_{\alpha}$ and $y-z=c_{0}<l$, (4) has at most one solution because $f\left(z+c_{0}\right)-f(z)$ increases with $z$. Hence $J_{l} \leq(l-1)\left[n^{\alpha}\right]$.

It then follows from (5) that

$$
J=\left[n^{\alpha}\right] n+\theta n^{3 \alpha}, \quad-1<\theta<1 .
$$

Now for a given $s \in f\left(A_{\alpha}\right) \pm f\left(B_{\alpha}\right)=T_{ \pm}$, we denote by $I_{ \pm}(s)$ the number of solutions of the equation $s=f(x) \pm f(v), x \in A_{\alpha}, v \in B_{\alpha}$. Then $\sum_{s \in T_{ \pm}} I_{ \pm}^{2}(s)=J$, $\sum_{s \in T_{ \pm}} I_{ \pm}(s)=\left[n^{\alpha}\right]\left(n-\left[n^{\alpha}\right]\right)$. Therefore using (6) we have

$$
\left[n^{\alpha}\right] n+\theta n^{3 \alpha}=J=\sum_{s \in T_{ \pm}} I_{ \pm}^{2}(s) \geq\left|T_{ \pm}\right|^{-1}\left(\sum_{s \in T_{ \pm}} I_{ \pm}(s)\right)^{2}=\left|T_{ \pm}\right|^{-1}\left[n^{\alpha}\right]^{2}\left(n-\left[n^{\alpha}\right]\right)^{2},
$$

that is, $\left|T_{ \pm}\right|\left(\left[n^{\alpha}\right] n+\theta n^{3 \alpha}\right) \geq\left[n^{\alpha}\right]^{2}\left(n-\left[n^{\alpha}\right]\right)^{2}$. Taking $\alpha=1 / 2$ we obtain the first estimate of our theorem. For $\alpha<1 / 2$, it follows that $\left|T_{ \pm}\right|>\left[n^{\alpha}\right] n-3 n^{3 \alpha}$ and therefore

$$
\left[n^{\alpha}\right] n-3 n^{3 \alpha}<\left|T_{ \pm}\right| \leq\left|f\left(A_{\alpha}\right) \pm f\left(A_{1}\right)\right| \leq\left[n^{\alpha}\right] n .
$$

Theorem 1.1 is proved.

REMARK. As an upper bound for $J_{l}$ we could use $(l-1)\left(\left[n^{\alpha}\right]-l\right)$. It would yield estimate (3) with any $c<3 / 4$ and sufficiently large $n>n_{1}(c)>0$ which we mentioned in Section 1. 


\section{Proof of Theorem 1.2}

All constants in this section may depend on $a$ and $\gamma$. Under the assumption that $n>n_{1}(a, \gamma)>0$ we put

$$
A=\left\{\left[n^{\alpha}\right]+1,\left[n^{\alpha}\right]+2, \ldots, 2\left[n^{\alpha}\right]\right\}, \quad B=\{[n / 2]+1,[n / 2]+2, \ldots, n\},
$$

where $\alpha=\gamma /(\gamma+1)$. In particular, the largest number in the set $A$ is less than the smallest number in the set $B$.

In analogy with Section 2 let $J$ be the number of solutions of the equation

$$
\left[a x^{\gamma}\right]+\left[a y^{\gamma}\right]=\left[a u^{\gamma}\right]+\left[a v^{\gamma}\right], \quad x, u \in A, \quad y, v \in B .
$$

Then

$$
J<n^{\alpha+1}+2 \sum_{1 \leq l<\left[n^{\alpha}\right]} J_{l},
$$

where $J_{l}$ denotes the number of solutions of the equation

$$
\left[a x^{\gamma}\right]+\left[a y^{\gamma}\right]=\left[a(x+l)^{\gamma}\right]+\left[a z^{\gamma}\right], \quad x, x+l \in A, \quad y, z \in B .
$$

Let us estimate $J_{l}$. Suppose that $x=x_{0} \in A$ is such a value of variable $x$ for which (8) has the largest number of solutions in terms of the variables $y, z$ but fixed $x$. Then $J_{l} \leq n^{\alpha} J_{l}^{\prime}$, where $J_{l}^{\prime}$ denotes the number of solutions in terms of the variables $y, z$ of the equation

$$
\left[a x_{0}^{\gamma}\right]+\left[a y^{\gamma}\right]=\left[a\left(x_{0}+l\right)^{\gamma}\right]+\left[a z^{\gamma}\right], \quad y, z \in B .
$$

From (9) it follows that $a y^{\gamma}-a z^{\gamma}<a\left(x_{0}+l\right)^{\gamma}-a x_{0}^{\gamma}+2$, that is,

$$
a(y-z) y_{1}^{\gamma-1}<2 a l x_{1}^{\gamma-1},
$$

where $x_{1}, y_{1}$ are some real numbers with $x_{0}<x_{1}<x_{0}+l, z<y_{1}<y$. Since $x_{0}+l \in A, z \in B$ then $0<y-z<c_{1} \ln ^{(\alpha-1)(\gamma-1)}$. Therefore,

$$
J_{l} \leq n^{\alpha} \sum_{m<c_{l} \ln ^{(\alpha-1)(\gamma-1)}} J_{l}^{\prime}(m)
$$

where $J_{l}^{\prime}(m)$ denotes the number of solution in one variable $z$ of the equation

$$
\left[a(z+m)^{\gamma}\right]-\left[a z^{\gamma}\right]=d, \quad z, z+m \in B,
$$

where $d=d(a, \gamma, n)$ is some fixed integer. 
Suppose that $z_{0}$ is the smallest solution of (11). Then

$$
a(z+m)^{\gamma}-a z^{\gamma}<a\left(z_{0}+m\right)^{\gamma}-a z_{0}^{\gamma}+4 .
$$

This inequality can be written as

$$
\gamma(\gamma-1) \int_{z_{0}}^{z} \int_{0}^{m} a(\phi+\psi)^{\gamma-2} d \phi d \psi<4 .
$$

Hence $0 \leq c_{2}\left(z-z_{0}\right) m n^{\gamma-2}<4$, that is, $J_{l}^{\prime}(m) \leq c_{3}\left(1+n^{2-\gamma} m^{-1}\right)$. In view of (10) we obtain thus

$$
J_{l} \underset{a, \gamma}{\ll} n^{\alpha}\left(l n^{(\alpha-1)(\gamma-1)}+n^{2-\gamma} \log n\right) .
$$

Taking (7) into account and $\alpha=\gamma /(\gamma+1)$ we obtain

$$
J \underset{a, \gamma}{\ll} n^{(2 \gamma+1) /(\gamma+1)}+n^{\left(2+3 \gamma-\gamma^{2}\right) /(\gamma+1)} \log n .
$$

In analogy with Section 2 set $T=\left\{\left[a x^{\gamma}\right]+\left[a y^{\gamma}\right]: x \in A, y \in B\right\}$. Obviously $|T| \leq|S+S|$. For a given $s \in T$, let $I(s)$ be the number of solutions of the representation $s=\left[a x^{\gamma}\right]+\left[a y^{\gamma}\right], x \in A, y \in B$. Then

$$
\begin{aligned}
& \sum_{s \in T} I^{2}(s)=J \underset{a, \gamma}{\ll} n^{(2 \gamma+1) /(\gamma+1)}+n^{\left(2+3 \gamma-\gamma^{2}\right) /(\gamma+1)} \log n, \\
& \sum_{s \in T} I(s)=\left[n^{\alpha}\right](n-[n / 2]) \underset{a, \gamma}{\gg} n^{(2 \gamma+1) /(\gamma+1)} .
\end{aligned}
$$

Hence

$$
|S+S| \geq|T| \geq \frac{\left(\sum_{s \in T} I(s)\right)^{2}}{\sum_{s \in T} I^{2}(s)} \underset{a, \gamma}{\gg \min }\left\{n^{1+\gamma /(\gamma+1)}, \frac{n^{\gamma}}{\log n}\right\} .
$$

Theorem 1.2 is proved.

Let us state two conjectures, the second of which is stronger than the first one.

CONJECTURE 3.1. For any $\epsilon>0,(\sqrt{5}+1) / 2<\gamma<2$, we have $|S+S| \underset{\epsilon, a, \gamma}{\gg} n^{\gamma-\epsilon}$.

CONJECTURE 3.2. For $3 / 2<\gamma<2|S+S| \underset{a, \gamma}{\gg} n^{\gamma}$.

It should be pointed out that if $\gamma=2$, then

$$
\frac{n^{2}}{\sqrt{\log n}} \underset{a}{\ll}|S+S| \underset{a}{\ll} \frac{n^{2}}{\sqrt{\log n}} .
$$


Indeed, Landau [9] showed that the number of integers not exceeding $N$ which can be expressed as a sum of two squares is asymptotic to $c N / \sqrt{\log N}$. Let $\omega=|S+S|$ and $t_{1}<t_{2}<\cdots<t_{\omega}$ be all different numbers of the set $S+S$. Then

$$
\left[a x_{j}^{2}\right]+\left[a y_{j}^{2}\right]=t_{j}, \quad j=1, \ldots, \omega, \quad 1 \leq x_{j} \leq n, \quad 1 \leq y_{j} \leq n .
$$

By taking $j_{m}=([a]+2) m, 1 \leq m \leq[\omega /([a]+2)]$ we obtain

$$
x_{j_{k+1}}^{2}+y_{j_{k+1}}^{2}>x_{j_{k}}^{2}+y_{j_{k}}^{2}+\frac{t_{j_{k+1}}-t_{j_{k}}-2}{a}>x_{j_{k}}^{2}+y_{j_{k}}^{2} .
$$

Therefore, $[\omega /([a]+2)] \ll n^{2} / \sqrt{\log n}$ from which the upper bound for $|S+S|$ follows. The lower bound is proved similarly.

\section{Acknowledgement}

The first author is highly indebted to the Institute of Mathematics, Academia Sinica, Taiwan for its warm hospitality and excellent working condition.

\section{References}

[1] J.-M. Deshouillers, 'Un probléme binaire en théorie additive', Acta Arith. 25 (1974), 393-403.

[2] G. Elekes, M. B. Nathanson and I. Z. Ruzsa, 'Convexity and sumsets', J. Number Theory 83 (2000), 194-201.

[3] M. Z. Garaev, 'On lower bounds for the $L_{1}$-norm of exponential sums', Math. Notes 68 (2000), 713-720.

[4] M. Z. Garaev and Ka-Lam Kueh, ' $L_{1}$-norms of exponential sums and the corresponding additive problem', Z. Anal. Anwendungen 20 (2001), 999-1006.

[5] S. A. Gritsenko, 'Three additive problems', Russian Acad. Sci. Izv. Math. 41 (1993), 447-464.

[6] N. Hegyvári, 'On consecutive sums in sequences', Acta Math. Acad. Sci. Hungar. 48 (1986), 193200.

[7] V. Jarnik, 'Über die Gitterpunkte auf konvexen Curven', Math. Z. 24 (1926), 500-518.

[8] S. V. Konyagin, 'An additive problem with fractional powers', Math. Notes 73 (2003), 594-597.

[9] E. Landau, 'Über die Einteilung der postiven ganzen zahlen in vier Klassen nach der Mindestzahl der zu ihrer additiven zusammen-setzung erforderlichen Quadrate', Arch. Math. Phys. 13 (1908), 305-312.

Instituto de Matemáticas UNAM

Institute of Mathematics

Campus Morelia

Academia Sinica

Ap. Postal 61-3 (Xangari)

Taipei 11529

CP 58089, Morelia, Michoacán

Nankang

México

Taiwan

e-mail: garaev@matmor.unam.mx

e-mail: maklk@ccvax.sinica.edu.tw 